\title{
On the Origin of the Slow Speed Solar Wind: Helium Abundance Variations
}

\author{
Cara E. Rakowski \& J. Martin Laming \\ Space Science Division, Naval Research Laboratory Code 7674L, Washington, D.C. 20375
}

\begin{abstract}
The First Ionization Potential (FIP) effect is the by now well known enhancement in abundance over photospheric values of Fe and other elements with first ionization potential below about $10 \mathrm{eV}$ observed in the solar corona and slow speed solar wind. In our model, this fractionation is achieved by means of the ponderomotive force, arising as Alfvén waves propagate through or reflect from steep density gradients in the solar chromosphere. This is also the region where low FIP elements are ionized, and high FIP elements are largely neutral leading to the fractionation as ions interact with the waves but neutrals do not. Helium, the element with the highest FIP and consequently the last to remain neutral as one moves upwards can be depleted in such models. Here, we investigate this depletion for varying loop lengths and magnetic field strengths.

Variations in this depletion arise as the concentration of the ponderomotive force at the top of the chromosphere varies in response to Alfvén wave frequency with respect to the resonant frequency of the overlying coronal loop, the magnetic field, and possibly also the loop length. We find that stronger depletions of $\mathrm{He}$ are obtained for weaker magnetic field, at frequencies close to or just above the loop resonance. These results may have relevance to observed variations of the slow wind solar He abundance with wind speed, with slower slow speed solar wind having a stronger depletion of He.
\end{abstract}

Subject headings: Sun:abundances - Sun:chromosphere - turbulence - waves

\section{Introduction}

Our knowledge of the solar wind has come a long way since the original prediction

of Parker (1958). It is now known to have two separate components; "fast wind" which originates in coronal holes with speeds generally greater than $600 \mathrm{~km} \mathrm{~s}^{-1}$, and "slow wind" with speeds less than $600 \mathrm{~km} \mathrm{~s}^{-1}$, the precise solar origin of which is uncertain. The fast 
solar wind is usually rather steady in speed and mass flux, and is generally taken to be the solar wind of Parker's prediction (Antiochos et al. 2011), although it is now recognized to be accelerated close to the sun by the damping of ion cyclotron and MHD waves (Ofman 2010) rather than by heat conduction as in the original concept (Parker 1963).

By contrast the slow solar wind is unsteady, with variability presumably reflecting solar source regions subject to varying phenomena associated with magnetic activity on the Sun. The slow solar wind is also distinguished from the fast by the properties of its turbulence. The fast wind exhibits essentially purely Alfvénic turbulence, whereas the slow wind also has magnetic structures advected within it (e.g. Bruno \& Carbone 2005). Since such structures may also be represented by superpositions of counter-propagating Alfvén waves in equal amplitude, turbulence in the slow wind is sometimes said to be "more balanced". The elemental composition of the two types of solar wind is also known to be different. Solar plasma in closed coronal loops and in the slow speed wind is known to be subject to a fractionation process, as the material that supplies these features moves up from the chromosphere to the corona. Elements which are strongly ionized in the chromosphere like Fe, $\mathrm{Si}, \mathrm{Mg}$, with first ionization potentials less than about $10 \mathrm{eV}$ (low FIP elements), are enhanced in abundance by a factor of about 3-4. High FIP elements are generally unaffected. He often shows a depletion in the solar corona (Laming \& Feldman 2001, 2003) and wind, which is larger and more variable in the slow wind (Aellig et al. 2001; Kasper et al. 2007) than in the fast (Reisenfeld et al. 2001). The abundance enhancement of low FIP ions in the fast solar wind is also much smaller than in the closed loop corona or the slow wind.

This variability arises naturally in models of the FIP effect (see Laming 2004, 2009, 2012, and discussed in more detail below), where the agent of fractionation is the ponderomotive force associated with Alfvén waves propagating between the chromosphere and the corona. The changing magnetic field geometry between open and closed field lines alters the propagation of Alfvén waves in such a way that the FIP fractionation produced by the ponderomotive force varies in the manner observed, i.e. more fractionation on closed field lines. Thus we assume henceforward that one fundamental difference between fast and slow solar wind is that slow solar wind must have originated on a closed coronal loop to become fractionated, and that this loop is subsequently opened up to release its plasma into wind.

Some authors argue that this distinction is not required. While the slow wind speed is well known to correlate inversely with the expansion rate of open field in equatorial coronal holes (Wang \& Sheeley 1990) leading to the association of the slow wind with super-radially expanding flux tubes at the edges of coronal holes (e.g. Ko et al. 2006), the view that the slow wind originates exclusively in open field regions has been advanced by Cranmer et al. (2007). They use their simulated Alfvén wave spectra in polar and equatorial coronal holes to 
calculate FIP fractionation following Laming (2004) and find a difference between fast wind from polar holes and slow wind from equatorial hole similar to that that is observed. On this basis, the transition in elemental composition from fast to slow wind should probably be gradual. However in observations (Geiss et al. 1995; Zurbuchen et al. 1999) this transition is abrupt, both in the change in composition and the freeze-in temperature associated with the O charge states. Antiochos et al. (2011) also comment that a tight correlation between element abundances and wind speed should result from such models, a finding which also is not supported by observation. We therefore adopt the view that the fast and slow winds are distinct entities, and the distinction arises as the fast wind originates exclusively in open field, while to become fractionated, the plasma comprising the slow wind must have spent time inside a closed coronal magnetic loop, which is subsequently opened up by reconnection.

In this paper we will investigate the abundance pattern expected from such a process. We follow Laming (2012) in assuming the Alfvén waves to have a coronal origin, presumably associated with heating mechanisms such as nanoflares and their associated reconnection (e.g. Sturrock 1999) or resonant absorption (e.g. Ruderman \& Roberts 2002). Due to repeated reflection at each loop footpoint (Ofman 2002), equal intensity Alfvén waves propagating in each direction are expected to be produced by these processes. We pay particular attention to variations of the abundance of helium, observed to change with slow solar wind speed (Aellig et al. 2001; Kasper et al. 2007). We show that not only does the FIP effect model based on the ponderomotive force (Laming 2004, 2009, 2012) explain the fact that the solar wind abundance of helium is lower than its generally accepted photospheric value, but that variations in this abundance may also be within reach of the model. While processes such as gravitational settling or inefficient Coulomb drag are not completely ruled out, their importance in forming the elemental composition of the solar wind is reduced from that discussed in previous works (Bochsler 2007; Kasper et al. 2007).

\section{The Ponderomotive Force Model}

In the model developed by Laming (2004, 2009, 2012), FIP fractionation has its origin in the action of the ponderomotive force on chromospheric ions, but not neutrals. The ponderomotive force is a second order term incorporating the $\rho \delta \mathbf{v} \cdot \nabla \delta \mathbf{v}$ and $\delta \mathbf{J} \times \delta \mathbf{B} / c$ terms in the MHD momentum equation. In many cases it looks like a force due to the gradient of wave magnetic pressure, but this obscures its true nature as the interaction of waves and particles through the refractive index of the plasma. Given this, it can be seen that both upward and downward ponderomotive accelerations may occur, with the latter giving a potential explanation for the so-called "inverse FIP" effect seen in active stars and 
in dwarfs of later spectral types than the sun (e.g. Wood \& Linsky 2010). The fractionation arises from the time averaged part of the ponderomotive force, which for waves of frequency $\omega_{A}<<\Omega$, the ion gyrofrequency, is given by (e.g Litwin \& Rosner 1998, Laming 2009)

$$
F=\frac{\partial}{\partial z}\left(\frac{q^{2} \delta E_{\perp}^{2}}{4 m \Omega^{2}}\right)=\frac{m c^{2}}{4} \frac{\partial}{\partial z}\left(\frac{\delta E_{\perp}^{2}}{B^{2}}\right)
$$

where $q$ is the ion charge. The ponderomotive acceleration, $F / m$, is independent of the ion mass.

Our basic approach builds on Hollweg (1984), and is illustrated by a cartoon in Figure 1. We start with a downward propagating Alfvén wave at the $\beta=1.2$ layer of one footpoint of a coronal loop, and integrate the non-WKB transport equations spatially for the forward and backward propagating Alfvén waves through the corona to the other footpoint, where we now have a mixture of waves propagating in both directions, depending on the resonant properties of the loop with respect to the wave frequency. The transport equations are (Cranmer \& van Ballegooijen 2005, and refs therein)

$$
\frac{\partial z_{ \pm}}{\partial t}+\left(u \pm V_{A}\right) \frac{\partial z_{ \pm}}{\partial r}=\left(u \pm V_{A}\right)\left(\frac{z_{ \pm}}{4 H_{D}}+\frac{z_{\mp}}{2 H_{A}}\right)
$$

where $z_{ \pm}=\delta v_{\perp} \pm \delta B_{\perp} / \sqrt{4 \pi \rho}$ are the Elsässer variables for Alfvén waves propagating against or along the magnetic field respectively. The Alfvén speed is $V_{A}=B / \sqrt{4 \pi \rho}$, the upward flow speed is $u$, the mass density is $\rho$, and the magnetic field is $B$. The signed scale heights are $H_{D}=\rho /(\partial \rho / \partial r)$ and $H_{A}=V_{A} /\left(\partial V_{A} / \partial r\right)$. In the solar chromosphere and corona $u<<V_{A}$, and we put $u=0$. Once $z_{ \pm}$are determined by numerical integration of equation 2 , we calculate $\delta v_{\perp}$ and $\delta B_{\perp} / \sqrt{4 \pi \rho}$, and hence the perpendicular electric field $\delta E_{\perp}$ from

$$
\begin{array}{cc}
\delta v_{\perp} & =\frac{z_{+}+z_{-}}{2} \\
\frac{\delta B_{\perp}}{\sqrt{4 \pi \rho}} & =\frac{z_{+}-z_{-}}{2} \\
\delta E_{\perp} & =\frac{\delta v_{\perp} B}{c}=\frac{\delta B_{\perp} V_{A}}{c} .
\end{array}
$$

Contributions from waves of the same frequency propagating in both directions along the magnetic field are naturally accounted for. In this work, for reasons of pedagogy, we only consider one pair of parallel forward and backward propagating Alfvén waves of a given frequency at a time, and neglect any nonlinear effects or dissipation. We are tacitly assuming that loop dynamics are mainly driven Alfvén wave interactions, as suggested by Bigot et al. (2008).

The degree of fractionation is given by the formula

$$
\text { fractionation }=\exp \left(2 \int_{z_{l}}^{z_{u}} \xi_{s} a\left(\nu_{e f f} / \nu_{s, i}\right) / v_{s}^{2} d z\right)
$$


(see equation 9, Laming 2009, equation 12, Laming 2004, which follow Schwadron et al. 1999), where $\xi_{s}$ is the ionization fraction of element $s, a$ is the ponderomotive acceleration, $\nu_{e f f}=\nu_{s, i} \nu_{s, n} /\left[\xi_{s} \nu_{s, n}+\left(1-\xi_{s}\right) \nu_{s, i}\right]$ where $\nu_{s, i}$ and $\nu_{s, n}$ are the collision rates of ions and neutrals, respectively, of element $s$ with the ambient gas. Also $v_{s}^{2}=k T / m_{s}+v_{\mu t u r b}^{2}+v_{\text {turb }}^{2}$, where $v_{\mu t u r b}$ is the amplitude of microturbulence coming from the chromospheric model, and $v_{\text {turb }}$ is the amplitude of longitudinal waves induced by the Alfvén waves themselves. These derive from the harmonic variation of the ponderomotive force, and unlike the fractionation, do not require an external density gradient. A full description of this is given in Laming (2012). The limits of integration, $z_{l}$ and $z_{u}$ bracket the region where the ponderomotive acceleration is effective in the chromosphere. Typically $z_{l}$ is at the $\beta=1.2$ layer in the low chromosphere, and $z_{u}$ is in the transition region where all elements are ionized.

The chromosphere at each footpoint can be based on any of the Vernazza, Avrett, \& Loeser (1981) models or similar. Here we use the Avrett \& Loeser (2008) update of VALC. The ionization balance of the minor ions is computed at each height in the chromosphere using the model temperature and electron density, and a coronal UV-X-ray spectrum appropriately absorbed in the intervening chromospheric layers. Laming (2012) investigated different approximations for computing the chromospheric ionization balance, and while the degree of FIP fractionation varied somewhat, the validity of this explanation for the phenomenon remained intact. The chromospheric magnetic field (illustrated in Figure 2) is taken to be a 2D force free field from Athay (1981) and designed to match chromospheric magnetic fields in Gary (2001) and Campos \& Mendes (1995), which represents the expansion of the field from the high $\beta$ photosphere where the field is concentrated into small network segments, into the low $\beta$ chromosphere where the field expands to fill much more of the volume. The coronal section of the loop has constant magnetic field, and a density scale height of 125,000 $\mathrm{km}$.

The precise model for the chromospheric magnetic field is not critical, because most of the FIP fractionation occurs in the upper chromosphere where the field is most uniform and vertical. In the lower chromospheric region with curving magnetic field in the plasma layer where sound and Alfvén speeds converge, contrary to the case in the corona, wave interaction processes play an important role. We do not attempt to model these directly, but argue that the increased longitudinal wave amplitudes in this region, arising from mode and parametric conversion, increase the value of $v_{s}^{2}$ in the denominator of the integrand in equation 4 to such an extent so as to render fractionation insignificant in this region. In Laming (2012), the longitudinal and Alfvén wave intensities are coupled by equation 15 in that reference, motivated by calculations illustrated in Cranmer et al. (2007) and Khomenko (2010). Here we note that recent work by Heggland et al. (2011) suggests that sound waves may be strong in this region even without the presence of Alfvén waves, as a result of turbulent motion in 
the convection zone.

\section{A Parameter Survey of FIP Fractionation}

In this paper we begin to explore the parameter space of magnetic field and loop length looking at coronal magnetic fields of $5 \mathrm{G}, 10 \mathrm{G}, 15 \mathrm{G}$, and loop lengths of 50,000 km, 75,000 $\mathrm{km}$, and $100,000 \mathrm{~km}$. Laming (2012) investigated the variation of FIP fractionation in a 100,000 km loop with 20G magnetic field, giving a loop resonance angular frequency of $0.07 \mathrm{rad} \mathrm{s}^{-1}$, as the Alfvén wave driver of the FIP effect varied with respect to this resonance. With wave amplitudes chosen to give a fractionation $\mathrm{Fe} / \mathrm{O}$ of about 4 as observed for example by Bryans et al. (2009), on resonance, He/O was about 0.6, S/O about 1.7, and $\mathrm{C} / \mathrm{O}$ about 1.2. As the wave frequency increased above the resonant value, as might happen for example if a coronal heating process excited waves at the loop resonance, followed by chromospheric evaporation reducing this resonant frequency, He/O decreased slowly at first and then increased up towards photospheric values, S/O increased rapidly then levels off to a maximum of $\sim 1.9$, and $\mathrm{C} / \mathrm{O}$ increased rapidly to a maximum of 1.6 and then began decreasing. Other fractionations varied also, but these three appeared the most robust and easiest to interpret in terms of available observational data.

Performing similar calculations for a wider variety of loop models, again keeping the $\mathrm{Fe} / \mathrm{O}$ fractionation to a value of about 4, we see a similar pattern with Alfvén wave frequency as was seen for $20 \mathrm{G}$ and 100,000 km. In Figure 3 we plot an example of how the fractionation varies with Alfvén wave frequency, for a field of $15 \mathrm{G}$ and a loop length of 75,000 km. Overlaid on the plot are the fractionations measured in the von Steiger et al. (2000) Ulysses survey of the solar wind. The color bar gives the range of frequencies examined, which start from just below resonance at $0.651 \mathrm{rad} \mathrm{s}^{-1}$. The black line is below resonance. The purple line is the resonant case. The two blue lines that still have strong helium depletion are just above resonance. The green, yellow and red lines are further from resonance, have less and less helium depletion and enhanced $\mathrm{S}$ and $\mathrm{C}$. In general the modeled fractionation pattern between $\mathrm{Mg}, \mathrm{Fe}$, and $\mathrm{Si}$ does not match the observations, with Fe predicted to have more FIP fractionation than actually observed in the solar wind. However other spectroscopic observations (Bryans et al. 2009) actually match this pattern very well (see Laming 2012).

In Figure 4, we plot the $\mathrm{He} / \mathrm{O}$ abundance ratio as a function of frequency, in units of the loop resonant frequency, for a variety of loop lengths and magnetic field strengths. The curve for 100,00 km loop length and $20 \mathrm{G}$ magnetic field is very similar to Laming (2012), with a minimum $\mathrm{He} / \mathrm{O}$ fractionation of 0.6 at resonance. The position of this minimum shows a clear trend to lower $\mathrm{He} / \mathrm{O}$ and higher frequency above resonance for weaker magnetic 
fields (and possibly also longer loop lengths); cases where the ponderomotive force is more concentrated at the top of the chromosphere. Figure 5 gives the coronal amplitude of the Alfvén wave in each case. For $5 \mathrm{G}$ and 100,000 km, and 10G with loop lengths 75,000 and $100,000 \mathrm{~km}$ it was not always possible to achieve a fractionation for $\mathrm{Fe} / \mathrm{O}$ of about 4 for any coronal wave amplitude, so these points were omitted in Figures 4 and 5 and in Figures 9 and 10 to follow below. The coronal wave amplitudes at resonance for each case are very similar, suggesting that if we normalized by this parameter instead of forcing $\mathrm{Fe} / \mathrm{O} \sim 4$, we would have very similar results. The curve for $15 \mathrm{G}, 75,000 \mathrm{~km}$ (the upper green curve) gives the coronal wave amplitudes for the fractionations shown in Figure 2.

Figure 6 shows the energy transmission characteristics of the various loops considered here, calculated from the fluxes of upgoing and downgoing waves in the loop footpoint encountered at the end of the numerical integration, and displayed with the same color coding as in preceding figures. On resonance, all loops have an energy transmission of unity. The width of this resonance increases with decreasing loop length, as does the minimum value of the energy transmission in between resonances. A trend with loop magnetic field is less easy to discern.

Figure 7 depicts the main features of the simulation for the case of $15 \mathrm{G}, 75,000 \mathrm{~km}$ and $0.07 \mathrm{rad} \mathrm{s}^{-1}$ just above the resonant frequency of $0.0651 \mathrm{rad}^{-1}$. Top left gives the density and temperature structure of the chromosphere to be read on the left (black) and right hand (gray) axes respectively. Bottom left gives the ponderomotive acceleration and slow mode wave amplitude. The "spike" in the ponderomotive acceleration can be seen to coincide with the steep density gradient at the top of the chromosphere. The ionization structure in the chromosphere, illustrated in the top right panel for $\mathrm{Fe}, \mathrm{Mg}, \mathrm{S}$, and $\mathrm{C}$, and also in the bottom right panel (gray lines, to be read on the right hand side axis) along with the FIP fractionations (black lines, to be read on the left axis), determines which elements can be accelerated and therefore fractionated.

$\mathrm{Fe}, \mathrm{Mg}, \mathrm{S}$ and $\mathrm{C}$ are all mostly ionized throughout the relevant region of the chromosphere, $\mathrm{H}$ and $\mathrm{O}$ become ionized between 1500 and $2000 \mathrm{~km}$ altitude in the chromosphere, and He is only ionized at the very top of the chromosphere. With our normalization of $\mathrm{Fe} / \mathrm{O}$ $\sim 4$, He becomes depleted relative to $\mathrm{O}$ when the ponderomotive acceleration is concentrated at the top of the chromosphere, i.e. when the "spike" associated with the steep density gradient is very strong, as in the case illustrated. $\mathrm{O}$ is accelerated up into the chromosphere while He is left behind, being the last element in the chromosphere to remain neutral. When waves penetrate deeper in the chromosphere $\mathrm{Fe}, \mathrm{Mg}, \mathrm{S}$ and $\mathrm{C}$ are all easily fractionated, and the relative contribution from the top of the chromosphere where $\mathrm{O}$ is accelerated and He left behind is less important. Since we are modeling specifically for $\mathrm{Fe} / \mathrm{O}$ of $4, \mathrm{He} / \mathrm{O}$ will 
be lowest when the acceleration region is confined to the top of the chromosphere, which happens close to the loop resonant frequency.

There are several factors that play into how deep waves penetrate into the chromosphere. Longer wavelength waves resonant with longer loops are not expected to penetrate so far into the chromospheric density gradients before being reflected back into the loop. This is clear from the fact that for a given loop, the first harmonic frequency is slightly less than twice that of the fundamental. Similar results are known for photospheric Alfvén waves launched upwards (Cranmer \& van Ballegooijen 2005; Verdini \& Velli 2007). However in our case, this effect is probably rather small, and not discernable on Figure 4 . Figure 8 illustrates this in more detail. The four panels show at top left the real (black) and imaginary (gray) parts of $\delta v_{\perp}$ (dashed lines) and $\delta B_{\perp} / \sqrt{4 \pi \rho}$ (solid lines), at bottom left the upgoing and downgoing wave energy fluxes, at top right the profile of the Alfvén speed, and at bottom right the profiles of $\partial V_{A} / \partial z$ (dashed lines) and $\sqrt{\left|V_{A} \partial^{2} V_{A} / \partial z^{2}\right|}$ (dash-dot line), again all for a wave of angular frequency $0.07 \mathrm{rad} \mathrm{s}^{-1}$ (horizontal dotted line in the bottom right panel) on a coronal loop with length $75,000 \mathrm{~km}$ and magnetic field $15 \mathrm{G}$. Expressions for cut-off frequencies, at which the Alfvén wave becomes evanescent, differ among various authors, but are of similar magnitude to quantities like $\partial V_{A} / \partial z$ and $\sqrt{\left|V_{A} \partial^{2} V_{A} / \partial z^{2}\right|}$. Also plotted as a solid line is $\sqrt{\left(\partial V_{A} / \partial z\right)^{2} / 4+\left|V_{A} \partial^{2} V_{A} / \partial z^{2}\right| / 2}$, which is given as the cutoff frequency by Musielak et al. (1992) and Moore et al. (1991). As can be seen, most wave reflection occuring at the steep chromospheric density gradient between 2100 and $2200 \mathrm{~km}$ altitude will not be greatly affected by changing of the Alfvén wave frequency in the range $0.01-0.1$ $\mathrm{rad} \mathrm{s}^{-1}$. Also, an increased wave frequency due to increased coronal magnetic field will not change its reflection properties, because the critical frequency also increases with $V_{A}$.

So while the energy transmission depends primarily on the loop length, and hence wavelength of the Alfvén wave, from Figure 4, it appears that the magnetic field is more important in controlling the degree of fractionation. Weaker magnetic field means that the altitude where the plasma $\beta \sim 1.2$ is higher, and the depth of chromosphere beneath a loop footpoint where fractionation may take place is reduced. The slow mode wave generation is also stronger in weaker magnetic fields, and so as the magnetic field becomes weaker, the more ponderomotive acceleration can be saturated by the associated longitudinal pressure. Additionally, compared to waves precisely on resonance, waves slightly off-resonance give a very similar ponderomotive acceleration profile, but generate more slow mode waves in lower regions of the chromosphere, again biasing fractionation further towards the top of the chromosphere. Thus we may expect the minimum He abundance to be just to higher frequency than the actual loop resonant frequency, as observed in our calculations. Further from resonance the spike in the ponderomotive force generates both positive and negative accelerations counteracting one another, such that essentially all fractionation occurs low 
down in the chromosphere, and He is thus not strongly depleted.

The importance in this example of the relative suppression of fractionation low in the chromosphere suggests that elements such as C or S might "echo" this behavior of He. As

explained in Laming (2012), these elements can be fractionated in the low chromosphere, assuming the ponderomotive acceleration is present here, because $\mathrm{H}$ is dominantly neutral. This means that elements fractionate roughly in proportion to their ion fraction, and both $\mathrm{C}$ and $\mathrm{S}$ are approximately 90\% ionized. Once $\mathrm{H}$ is ionized, fractionation is suppressed unless the charge state fraction is much closer to 1 ; for example Fe and $\mathrm{Mg}$ have ionization fractions of 0.9995 and 0.998 respectively. Thus when the ponderomotive acceleration is concentrated at the top of the chromosphere, and when He is strongly depleted, S and $\mathrm{C}$ should have relatively little fractionation, and correspondingly when the acceleration is present in the low chromosphere and $\mathrm{He} / \mathrm{O}$ is relatively unaffected, $\mathrm{S}$ and $\mathrm{C}$ should display some fractionation.

Figures 9 and 10 show $\mathrm{C} / \mathrm{O}$ and $\mathrm{S} / \mathrm{O}$ as a function of frequency. Both show the expected qualitative behavior with frequency and magnetic field, although the minimum in these ratios appears always to be at the loop resonance, as a steeply peaked dip. A small distance above resonance the fractionation is a function of magnetic field and loop length with the highest fractionations for the highest magnetic field and the shorter loop lengths. The fractionation peaks at some value and then declines far away from resonance.

\section{Variation of the FIP Effect with Solar Activity?}

\subsection{The Helium Abundances}

Aellig et al. (2001) and Kasper et al. (2007) both found that the solar wind Helium abundance varied with wind speed and the solar cycle. Although there is a large scatter in the individual data points (Aellig et al. 2001) the He/H ratio averaged over a velocity bin increases almost monotonically with wind speed. The variation with wind speed is wider during solar minimum than during solar maximum in the sense that the strongest average He depletion is seen at solar minimum.

We calculate fractionations relative to $\mathrm{O}$, on the assumption that all elements can be treated as minor ions (this is not true for $\mathrm{H}$ ). To be certain that this did not compromise our results we looked at the publicly available level 2 ACE SWICS He/O and He velocity data over the life of the mission so far. Figure 11 reproduces the findings of Aellig et al. (2001) and Kasper et al. (2007) with respect to wind speed, in that lower He velocities correspond to lower average He/O ratios in a mostly monotonic fashion. We do not find the strong 
dependence of the helium abundance on phase of the solar cycle. It must be remembered that we are considering He/O, whereas Aellig et al. (2001) and Kasper et al. (2007) worked with $\mathrm{He} / \mathrm{H}$.

These authors followed several antecedents and discussed their results in terms of inefficient Coulomb drag or gravitational settling. We are going to argue that this abundance anomaly is part of the same process that gives rise to the FIP effect, but before doing so we briefly review this alternative. Gravitational settling is known to occur on the Sun in quiescent streamers (Feldman et al. 1998), where Fe is observed to be depleted relative to other elements. Noci et al. (1997), following Geiss et al (1970), make the point that if such processes were the origin of the solar wind He abundance depletion, other heavy ions should also be depleted, which is now known not to be the case. Bochsler (2007) revisits this, comparing $\mathrm{He}, \mathrm{O}$, and $\mathrm{Ne}$ for which He appears most affected by gravitational settling, or inefficient Coulomb drag, in the corona. In this case the He abundance should correlate with the proton flux, which is not observed (Wang 2008). He and other minor ions are also known to flow faster than $\mathrm{H}$ in the solar wind observed in situ at $1 \mathrm{AU}$ (e.g. Neugebauer et al. 1996; Kasper et al. 2008; Bourouaine et al. 2011) which conflicts with the notion of a proton driven wind dragging the rest of the minor ions out with it, unless a second stage of wind acceleration is invoked further out in the heliosphere (Wang 2008). Laming \& Feldman (2003) also measure a depleted He abundance in coronal hole and quiet solar region at altitudes below that where He is presumed to be depleted in Bochsler (2007).

We believe we can better understand the variation of the solar wind He abundance in the context of our model of the FIP effect. Figure 4 shows the variation of $\mathrm{He} / \mathrm{O}$ as a function of the frequency relative to the resonant frequency. The strongest He depletions are only seen just above the resonant frequency. He/O approaches its photospheric value as one moves further away from resonance. Consider a loop being heated by a process that also generates Alfvén waves. So long as the perturbation to the magnetic field is small compared to the loop initial magnetic field, only the resonant frequencies of the loop would be excited. However the heating would evaporate material from the footpoints of the loop up into the loop, changing the resonant frequency. Low levels of activity and heating would only change the resonance by a small amount. More active regions would have greater heating, greater evaporation and thus move the loop further away from the original resonance. The He depletion due to the originally excited waves then depends on how far from resonance the loop became. Thus according to our parameter search, higher slow wind speeds should be associated with plasma that was originally in (shorter) loops with higher magnetic field. 


\subsection{The Sulfur and Carbon Abundances}

In the above picture of loop excitation, we always expect the excited wave to be slightly off resonance due to chromospheric evaporation. Thus from figures 9 and 10 we expect the S and $\mathrm{C}$ fractionations to be a function primarily of the magnetic field in the loop. This means we predict that one should see the average $\mathrm{C}$ and $\mathrm{S}$ varying with solar cycle and wind speed, just as is seen for He. While $\mathrm{S}$ is not available in the level 2 ACE products we can test this hypothesis for C. In figure 12, we plot the average C/O relative to solar (0.550 Asplund et al. 2009) for 9 velocity bins as a function of time, in the same way as figure 11 for He/O. For at least the years 2000 to 2004 and during 2005 a variation with wind speed is apparent, with the slowest wind speeds showing a lower $\mathrm{C} / \mathrm{O}$ fractionation. The period around 2003 also corresponds to a period of strong He/O fractionation with wind speed. This fits well with the prediction of our model. Further confirmation could be found if S/O could be analyzed as well. A similar correlation between $\mathrm{He} / \mathrm{O}$ and $\mathrm{C} / \mathrm{O}$ has been found (Ko et al. 2006, see their Figure 11), who analyzed ACE Level 2 data collected over a week timescale as fast wind from an equatorial coronal hole transitions to slow wind from the surrounding corona.

\subsection{The Coronal Origin of Alfvén Waves}

Our finding that Alfvén waves on resonance with a coronal loop give FIP fractionations in better agreement with observations than other frequencies reinforces our suggestion of a coronal origin for the waves. No chromospheric origin would naturally achieve such selectivity, unlike the expectation from a coronal origin. For example, consider a flux tube with cross-B density gradient scale length $l$, undergoing a kink mode oscillation which become Alfvénic at a resonant surface where the wave frequency $\omega=2 L / V_{A}$, where $L$ is the loop length and $V_{A}$ is the Alfvén speed (see e.g. Ruderman \& Roberts 2002). The width of this resonant layer is $\delta \sim(l \nu / \omega)^{1 / 3}$, where $\nu=a V_{A} / R$ is the kinematic viscosity, $a$ is the loop radius and $R$ is the Reynolds number. The Alfvénic velocity fluctuations are larger than those of the kink mode by a factor $l / \delta=\left(l^{2} R / a L\right)^{1 / 3} \sim 0.1 R^{1 / 3}$ under typical conditions, and are constrained to be resonant with the flux tube and their location of excitation.

The nanoflare paradigm suggests that the dominant loop footpoint motions are of much lower frequency, and do not excite oscillations in the loop but act so as to build up magnetic stresses in the corona. These stresses periodically release themselves, in what has become known as a "nanoflare", as a current sheet develops. Rappazzo et al. (2007, 2008) discuss the buildup of magnetic stresses within the framework of turbulence phenomenology, where it appears that velocity perturbations similar to the $\sim 30 \mathrm{~km} \mathrm{~s}^{-1}$ observed should be expected. Longcope et al. (2009) conjecture that in impulsive reconnection in post-flare loops, 
only about $10 \%$ of the liberated magnetic energy is converted directly into heat, the rest reappearing as kinetic energy that ultimately drives turbulence. So long as the perturbation to the magnetic field is sufficiently smaller than the ambient magnetic field, the boundary conditions imposed by the loop footpoints will restrict the modes excited by the reconnection event to those that are resonant with the loop.

Sturrock (1999) gives a pedagogic review of the mechanisms by which various wave modes may be excited by reconnection. The reconnected field line is generally distorted, and this can either propagate away from the reconnection site as an Alfvén wave, or emit magnetoacoustic waves traveling perpendicularly to the magnetic field direction. Kigure et al. (2010) explicitly consider the generation of Alfvén waves by magnetic reconnection, and find that a significant fraction of the magnetic energy released (several tens of $\%$, depending on geometry and plasma $\beta$ ) can be carried off by Alfvén or magnetoacoustic (fast or slow mode) waves, with Alfvén waves dominating for $\beta<1$. Liu et al. (2011) discuss the role of temperature anisotropies and wave generation by the firehose instability in the outflow.

\section{Origin of Slow Speed Solar Wind}

Based on the variation on the helium abundance in the slow speed wind wind, we argue that the faster slow speed wind should have originated in magnetic flux tubes with higher magnetic field, with the slower components of the slow wind arising from flux tubes with weaker magnetic field. Slow wind speed is known to correlate with the expansion rate of the open field (Wang \& Sheeley 1990). Wang \& Sheeley (2003) argue that at sunspot maximum, more slower speed slow wind is produced, and that this comes mainly from small sheared open field regions located near active regions. These have relatively high footpoint field strengths, and correspondingly high expansion factors. The high coronal heating rate near the footpoint (heating is presumed to correlate with magnetic field strength) increases the solar wind density, thereby reducing its speed for the same energy input. Our inference concerns not this open field considered by Wang (2008) and Bochsler (2007), but the closed field with which it presumably reconnects releasing plasma into the solar wind.

Attempts have been made to map in situ slow solar wind observations by Ulysses and ACE spacecraft back to a magnetic field source surface at a heliocentric distance of around 2.5 $R_{\odot}$, and thence back to the solar disk itself (Neugebauer et al. 2002; Liewer et al. 2004), with the result that at sunspot maximum (but not at activity minimum), open field lines in active regions are the most likely source. Wang (2008) studied the solar wind helium abundance and found that it correlated best with the source-surface magnetic field strength, which was found to be strong in fast wind and slow wind at sunspot maximum, but weak in the low-speed 
solar wind emanating from coronal hole boundaries at solar minimum. Increased proton flux in the low-speed solar wind should increase the He abundance if inefficient Coulomb drag is important, but as mentioned above, Wang (2008) found essentially no correlation between these variables.

If the magnetic field in the originally closed loop correlates with the strength of neighboring open field with which it must reconnect, our conclusions match these results. From Figure 3, the dependence of $\mathrm{He} / \mathrm{O}$ on magnetic field for a given loop length appears to be much stronger than the dependence on loop length for a given magnetic field, although there is a tendency for longer loops to have stronger helium depletions.

Our results strongly suggest that the solar wind helium abundance is set in the chromosphere, along with the other abundance modifications that result in the FIP effect. Byhring (2011) reaches a similar conclusion, but argues that it must arise from gravitational settling. However such processes; diffusion, gravitational settling and inefficient Coulomb drag are too slow to have an appreciable effect in a solar atmosphere that is increasingly being appreciated as a dynamic environment. For example timescales in diffusion models range from tens of hours (Hansteen et al. 1994) to days or weeks (Killie \& Lie-Svendsen 2007), and so such possibilities must be considered highly implausible.

Our calculations necessarily have approximations in the treatment of wave physics (see Laming 2012, for fuller discussion). However all of the phenomena we discuss have qualitative explanations in terms of the structure of the chromosphere and its ionization balance. While absolute numbers for fractionations may vary slightly in future works as chromospheric models and atomic physics improve, we expect that the various correlations between element abundances and the relation of the FIP fractionation to the wave frequency in terms of the loop resonant frequency to be robust. Most of the variations we have discussed center around the "spike" in the ponderomotive acceleration at the top of the chromosphere where the density gradient is steep. At first sight it might seem a coincidence that this "spike" occurs where the hydrogen in the chromosphere is becoming ionized, and thus allows for FIP fractionation. This is not the case. The steep density gradient arises precisely because hydrogen is becoming ionized, and so cooling by radiation in Lyman $\alpha$ is becoming quenched. Consequently heat input into the plasma cannot be radiated away, and the temperature must rise. The density decreases to maintain constant pressure, and waves in this region give rise to the strong ponderomotive acceleration.

This work was supported by NASA Contracts NNH10A055I, NNH11AQ23I, and by basic research funds of the Office of Naval Research. We are grateful to Dan Moses for a careful reading of the paper. 


\section{REFERENCES}

Aellig, M. R., Lazarus, A. J., \& Steinberg, J. T. 2001, GRL, 28, 2767

Antiochos, S. K., Mikić, Z., Titov, V. S., Lionello, R., \& Linker, J. A. 2011, ApJ, 731, 112

Asplund, M., Grevesse, N., Sauval, A. J., \& Scott, P. 2009, ARA\&A, 47, 481

Athay, R. G. 1981, ApJ, 249, 340

Avrett, E., \& Loeser, R. 2008, ApJS, 175, 229

Bigot, B., Galtier, S., \& Politano, H. 2008, A\&A, 490, 325

Bochsler, P. 2007, A\&A, 471, 315

Bourouaine, S., Marsch, E., \& Neubauer, F. M. 2011, ApJ, 728, L3

Bruno, R., \& Carbone, V. 2005, Living Rev. Solar Phys. 2, 4

Bryans, P., Landi, E., \& Savin, D. W. 2009, ApJ, 691, 1540

Byhring, H. S. 2011, ApJ, 738, 172

Campos, L. M. B. C., \& Mendes, P. M. V. M. 1995, MNRAS, 276, 1041

Cranmer, S. R., \& van Ballegooijen, A. A. 2005, ApJS, 156, 265

Cranmer, S. R., van Ballegooijen, A., \& Edgar, R. J. 2007, ApJS, 171, 520

Feldman, U., Schühle, U., Widing, K. G., \& Laming, J. M. 1998, ApJ, 505, 999

Gary, G. A. 2001, Solar Physics, 203, 71

Geiss, J., Gloeckler, G., \& von Steiger, R. 1995, Space Science Reviews, 72, 49

Geiss, J., Hirt, P., \& Leutwyler, H. 1970, Solar Physics, 12, 458

Hansteen, V. H., Leer, E., \& Holzer, T. E. 1994, ApJ, 428, 843

Heggland, L., Hansteen, V. H., De Pontieu, B., \& Carlsson, M. 2011, ApJ, 743, 142

Hollweg, J. V. 1984, ApJ, 277, 392

Huba, J. D., Warren, H.P., Joyce, G., Pi, X., Iijima, B., \& Coker, C. 2005, GRL,32, L15103 
Kasper, J. C., Stevens, M. L., Lazarus, A. J., Steinberg, J. T., \& Ogilvie, K. W. 2007, ApJ, 660,901

Kasper, J. C., Lazarus, A. J., \& Gary, S. P. 2008, Phys. Rev. Lett., 101, 261103

Khomenko, E. 2010, in Highlights of Spanish Astrophysics V, Astrophysics and Space Science Proceedings, (Spring-Verlag: Berlin Heidelberg), p 51

Kigure, H., Takahashi, K., Shibata, K., Yokoyama, T., \& Nozawa, S. 2010, PASJ, 62, 993

Killie, M. A., \& Lie-Svendsen, Ø. L. 2007, ApJ, 666, 501

Ko, Y.-K., Raymond, J. C., Zurbuchen, T. H., Riley, P., Raines, J. M., \& Strachan, L. 2006, ApJ, 646, 1275

Liewer, P. C., Neugebauer, M., \& Zurbuchen, T. 2004, Solar Physics, 223, 209

Laming, J. M., \& Feldman, U. 2001, ApJ, 546, 552

Laming, J. M., \& Feldman, U. 2003, ApJ, 591, 1257

Laming, J. M. 2004, ApJ, 614, 1063

Laming, J. M. 2009, ApJ, 695, 954

Laming, J. M. 2012, ApJ, 744, 115

Litwin, C., \& Rosner, R. 1998, ApJ, 506, L143

Liu, Y.-H., Drake, J. F., \& Swisdak, M. 2011, Phys. Plasmas, 18, 2102

Longcope, D. W., Guidoni, S. E., \& Linton, M. G. 2009, ApJ, 690, L18

Moore, R. L., Musielak, Z. E., Suess, S. T., \& An, C.-H. 1991, ApJ, 378, 347

Musielak, Z. E., Fontenla, J. M., \& Moore, R. L. 1992, Phys. Fluids B, 4, 13

Neugebauer, M., Liewer, P. C., Smith, E. J., Skoug, R. M., \& Zurbuchen, T. H. 2002, J. Geophys. Res., 107, 1488

Neugebauer, M., Goldtein, B. E., Smith, E. J., \& Feldman, W. C. 1996, J. Geophys. Res., 101, 17047

Noci, G., et al. 1997, ESASP, 404, 75

Ofman, L. 2010, Living Rev. Solar Phys., 7, 4 
Ofman, L. 2002, ApJ, 568, L135

Parker, E. N. 1958, ApJ, 128, 669

Parker, E. N. 1963, Interplanetary Dynamic Processes (New York: Interscience)

Rappazzo, A. F., Velli, M., Einaudi, G., and Dahlburg, R. B., 2007, ApJ, 657, L47

Rappazzo, A. F., Velli, M., Einaudi, G., and Dahlburg, R. B., 2008, ApJ, 677, 1348,

Reisenfeld, D. B., Gary, S. P., Gosling, J. T., Steinberg, J. T., MacComas, D. J., Goldstein, B. E., \& Neugebauer, M. 2001, J. Geophys. Res., 106, 5693

Ruderman, M. S., \& Roberts, B. 2002, ApJ, 577, 475

Schwadron, N. A., Fisk, L. A., \& Zurbuchen, T. H. 1999, ApJ, 521, 859

Sturrock, P. A. 1999, ApJ, 521, 451

Verdini, A., \& Velli, M. 2007, ApJ, 662, 669

Vernazza, J., \& Reeves, E. M. 1978, ApJS, 37, 485

Vernazza, J., Avrett, E. H., \& Loeser, R. 1981, ApJS, 45, 635

von Steiger, R., et al. 2000, J. Geophys. Res., 105, 27217

Wang, Y.-M., \& Sheeley, N. R., Jr. 1990, ApJ, 355, 726

Wang, Y.-M., \& Sheeley, N. R., Jr. 2003, ApJ, 587, 818

Wang, Y.-M. 2008, ApJ, 683, 499

Wood, B. E., \& Linsky, J. L. 2010, ApJ, 717, 1279

Zurbuchen, T. H., Hefti, S., Fisk, L. A., Gloeckler, G., \& von Steiger, R. 1999, Space Science Reviews, 87, 353 


\section{corona}

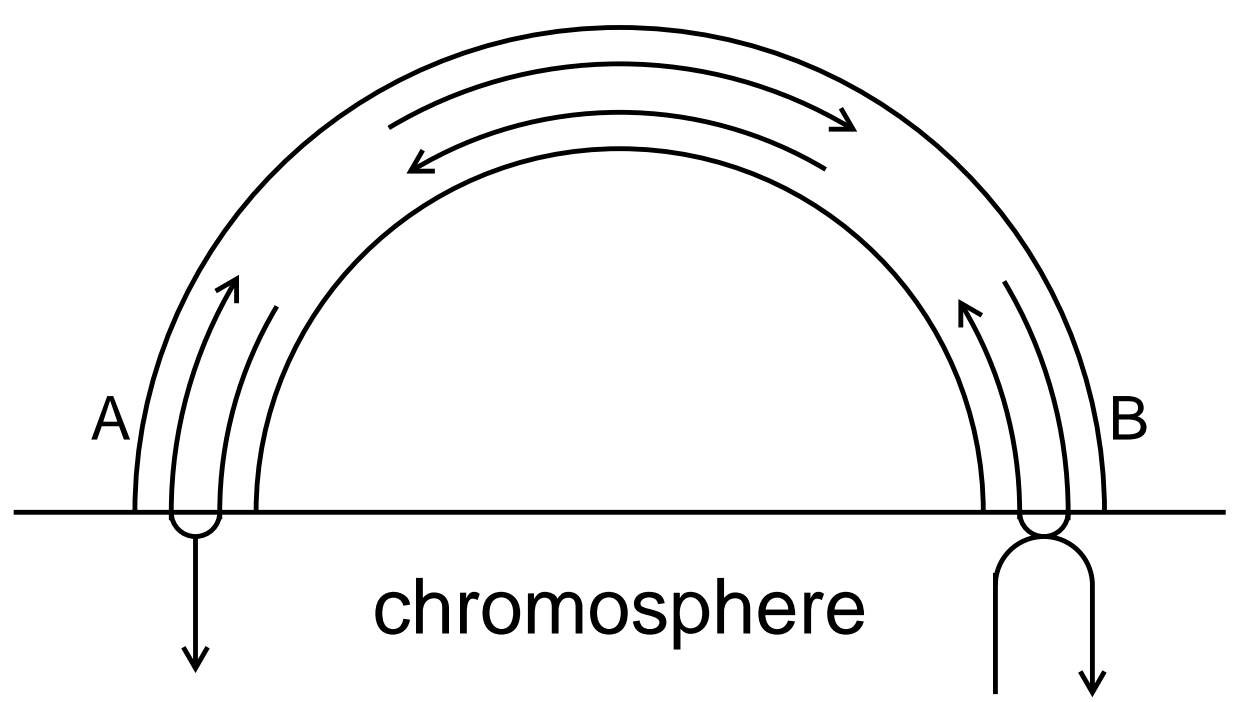

Fig. 1.- Cartoon illustrating the model. The integration of the Alfvén wave transport equations is begun in chromosphere A with one downgoing wave. Integrating back through the corona to chromosphere $\mathrm{B}$, forward and backward propagating waves develop. At chromosphere $\mathrm{B}$, the wave is either reflected back into the corona, or transmitted into the chromosphere, depending on the wave frequency with respect to the resonant frequency of the loop. Resonant waves reflect, nonresonant waves precipitate down. 


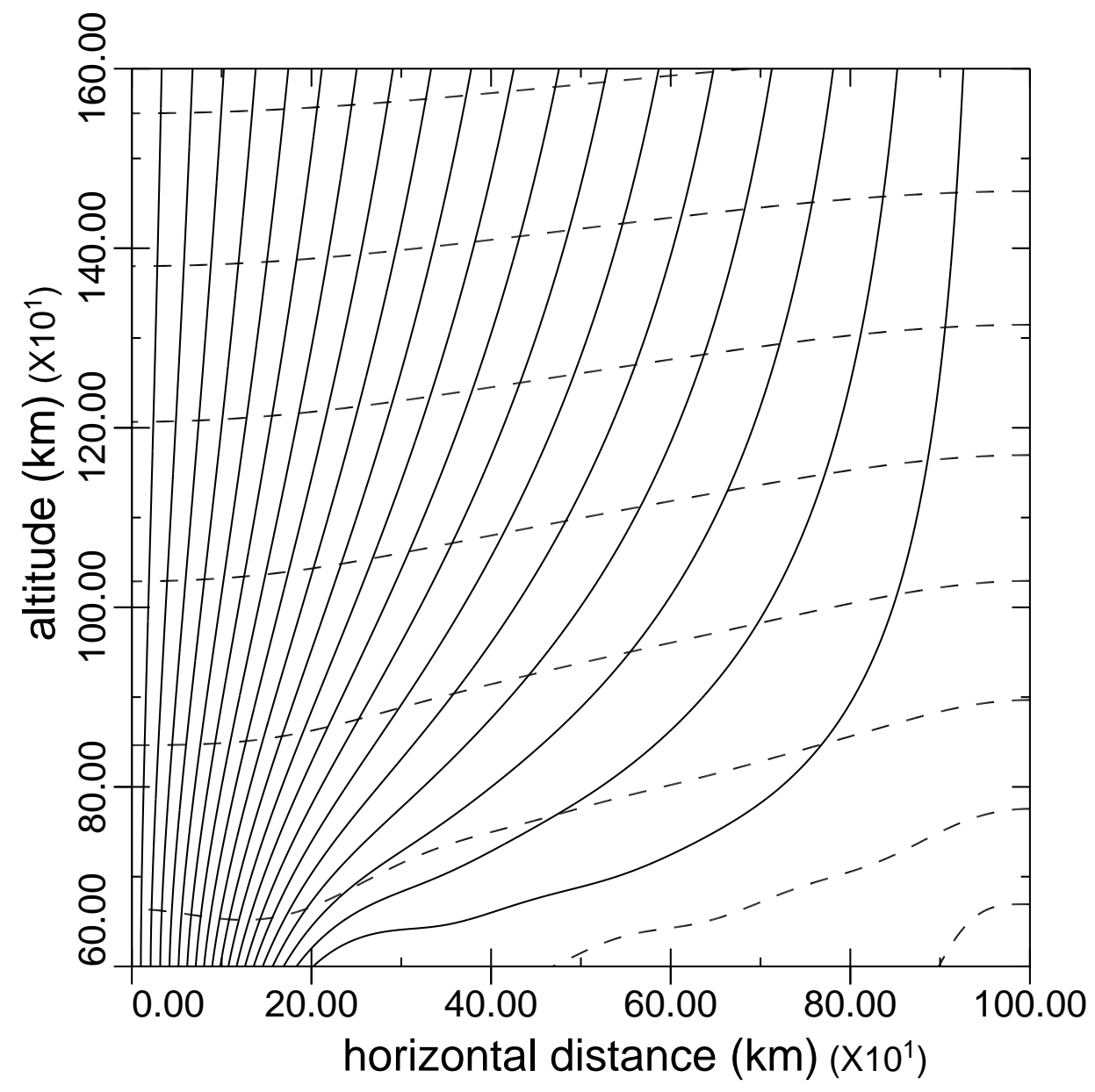

Fig. 2.- Depiction of force-free chromospheric magnetic field for coronal loop model with $B=15 \mathrm{G}$, calculated from Athay (1981). Solid lines indicate magnetic field lines. Dashed line indicate contours of the Alfvén speed. The plasma $\beta=1.2$ layer is at $700 \mathrm{~km}$ altitude above the photosphere, and moves up and down for weaker or stronger coronal fields respectively. 


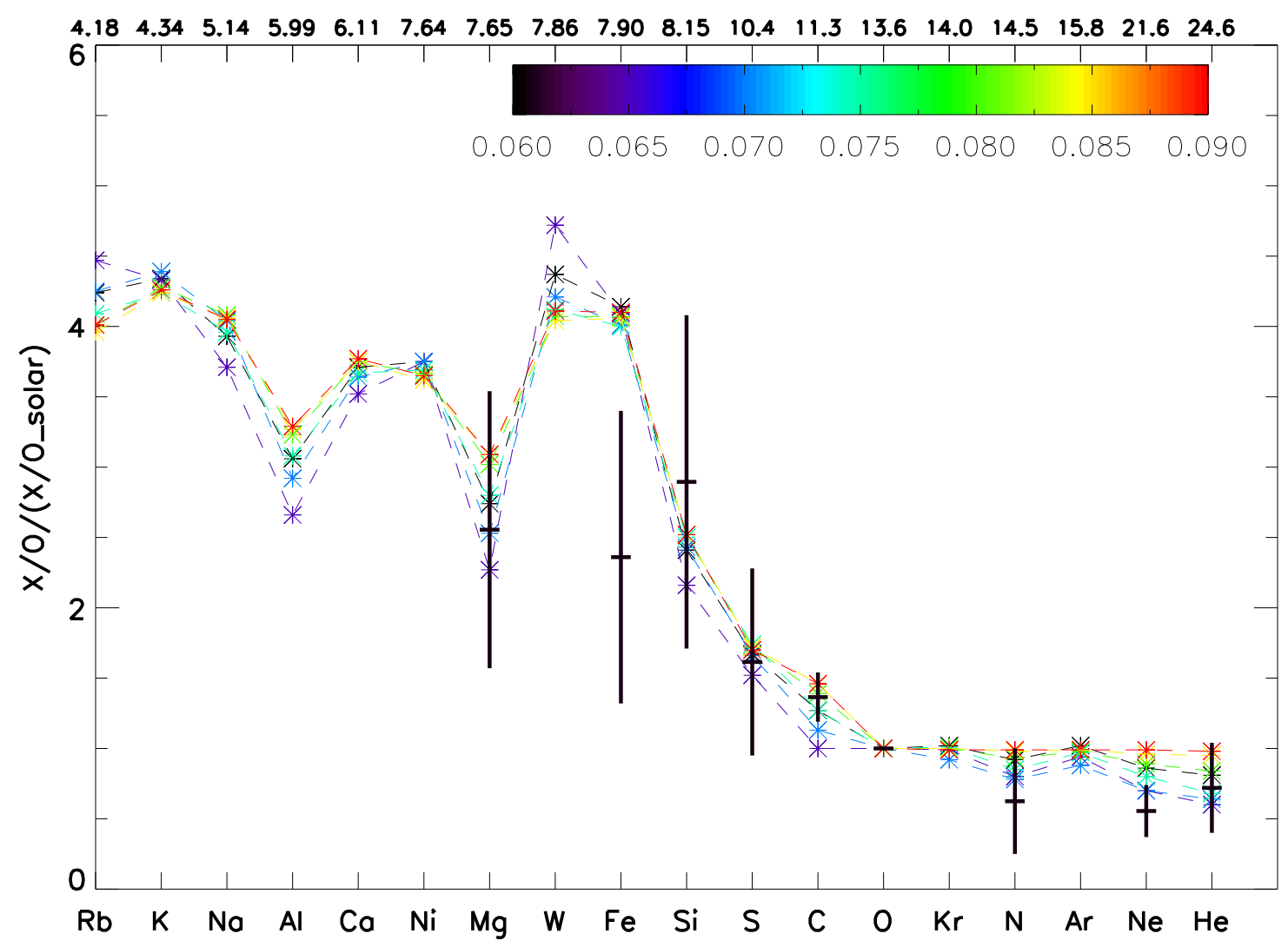

Fig. 3.- Fractionations relative to oxygen relative to their photospheric values as a function of FIP for the case of $15 \mathrm{G}$ and $75,000 \mathrm{~km}$. Overlaid are the measured fractionations of the elements included in von Steiger et al. 2000. The strongest He depletion corresponds to the purple line, illustrating the resonant case. As one moves off resonance, the He abundance increases. Other elements are predicted to vary either in correlation (e.g. $\mathrm{Al}, \mathrm{Mg}, \mathrm{S}, \mathrm{C}$ ), or anti-correlation (e.g. Rb, W) with He. 


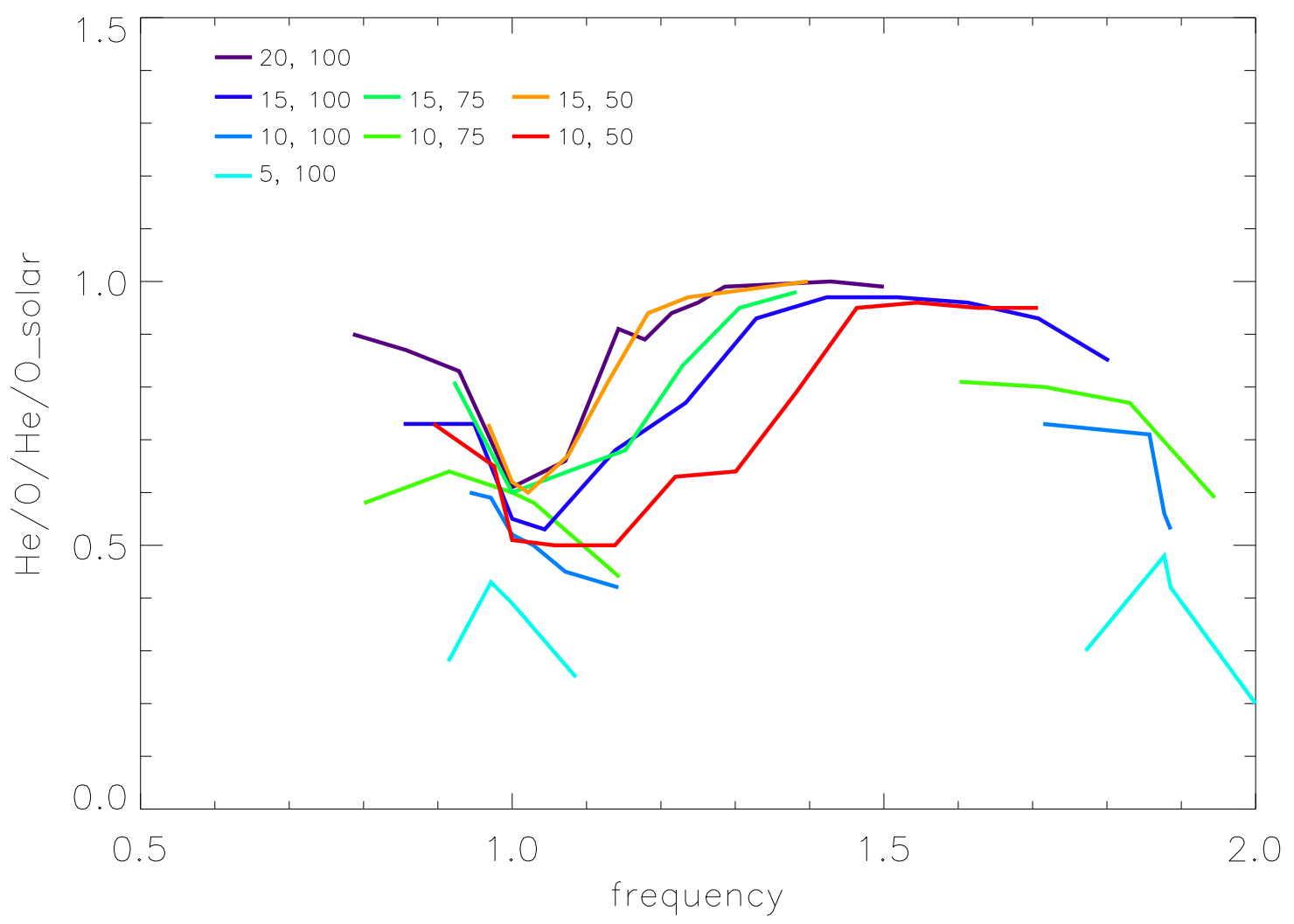

Fig. 4. - He/O relative to their solar ratio as a function of the frequency divided by the resonance frequency. For $5 \mathrm{G}$ and $100,000 \mathrm{~km}$ the points where it was not possible to obtain $\mathrm{Fe} / \mathrm{O}$ of 4 for any amplitude, and these point are omitted. For $10 \mathrm{G}$ and 75,000 or 100,000 $\mathrm{km}$, it was also not possible to achieve $\mathrm{Fe} / \mathrm{O} \sim 4$ for any amplitude, and these points are also omitted. A trend of increasing He depletion with decreasing magnetic field and possibly also increasing loop length can be seen. 


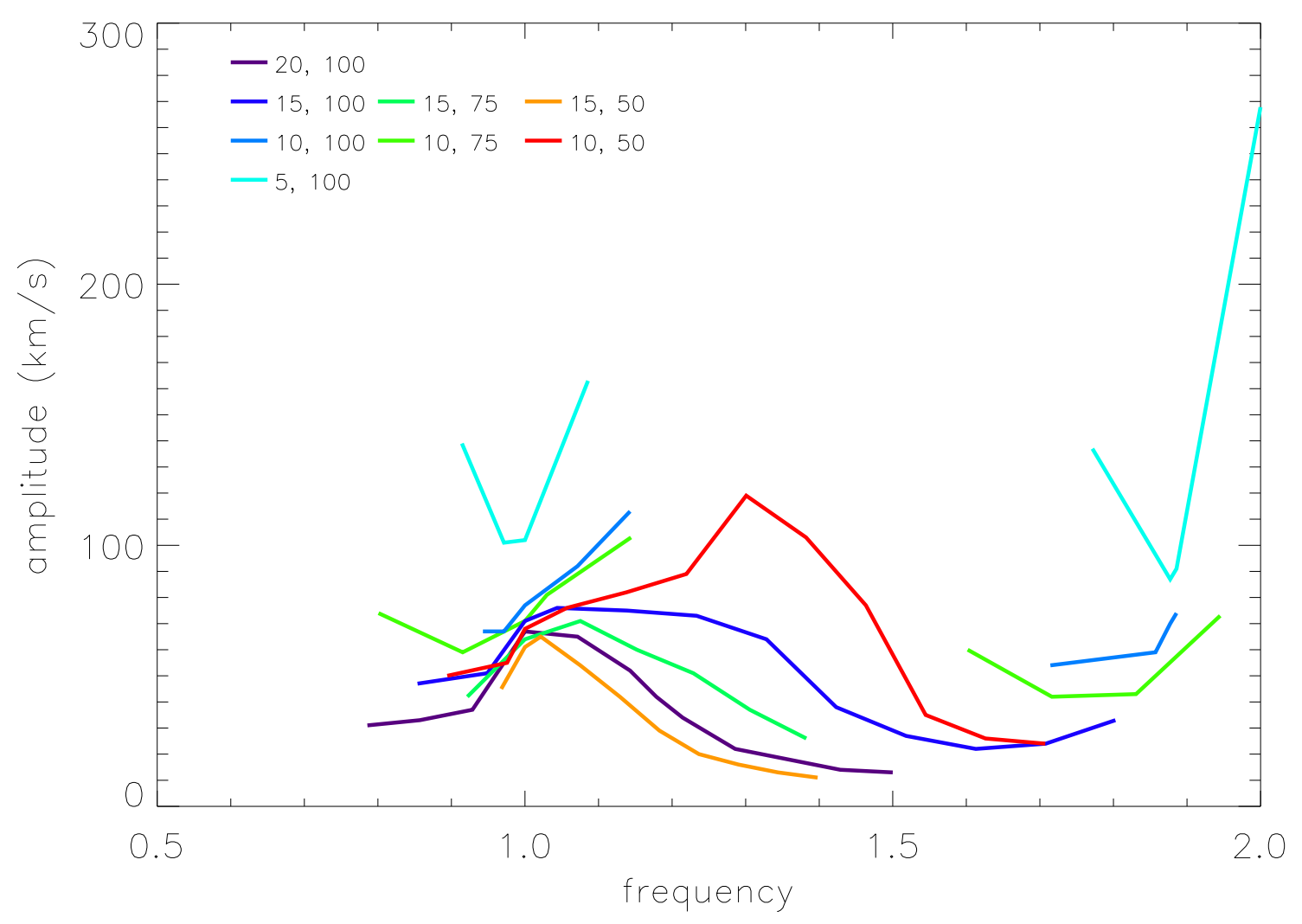

Fig. 5.- Amplitude in $\mathrm{km} \mathrm{s}^{-1}$ at the peak of the loop as a function of frequency relative to the resonance frequency. The absent points have the same meaning as in Figure 3. All curves are selected to give $\mathrm{Fe} / \mathrm{O}$ close to 4 , but as can be seen, close to resonance, this results in very similar wave amplitudes, even though loop lengths and background magnetic fields are varying. 


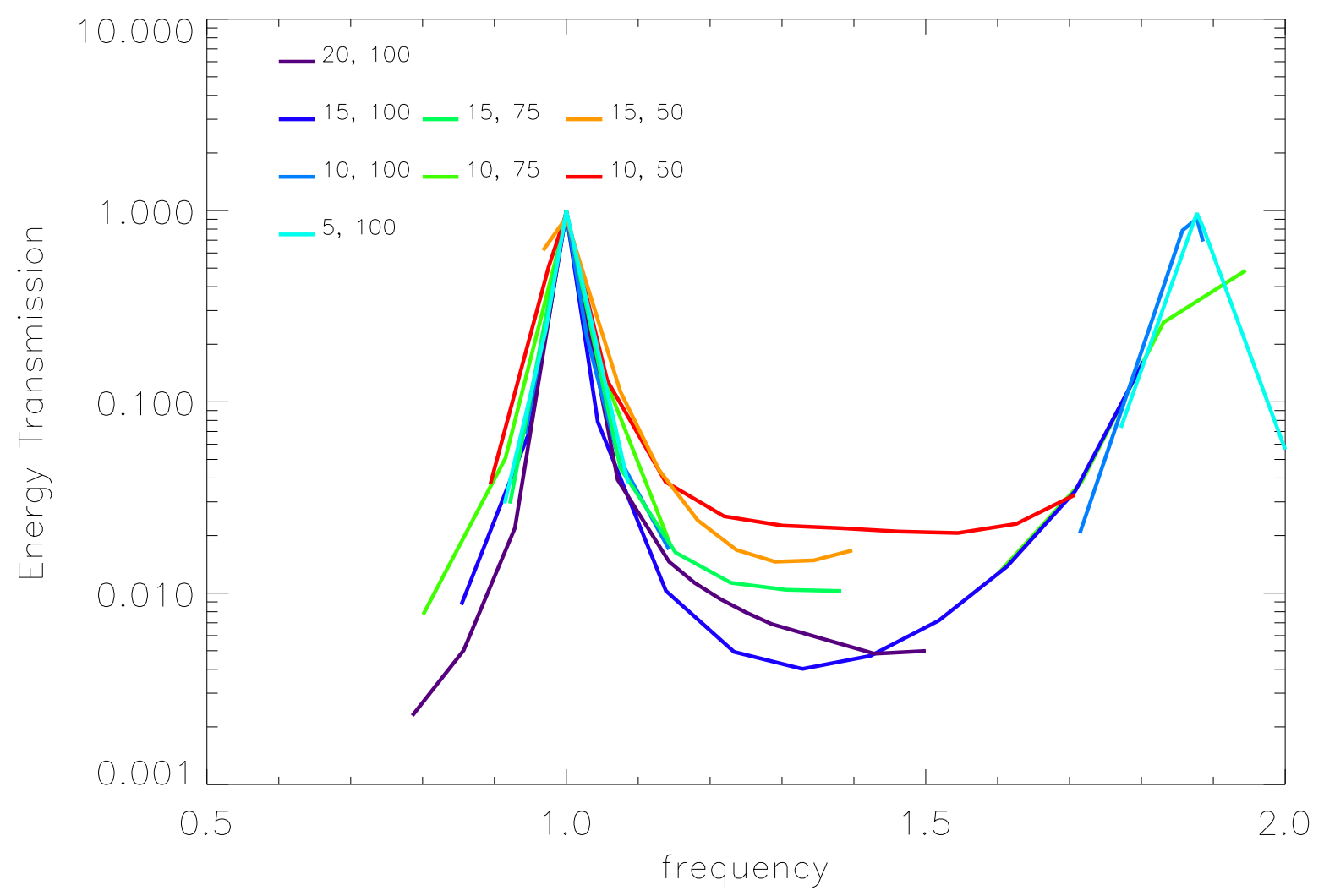

Fig. 6.- The energy transmission coefficient as a function of wave frequency (expressed in terms of the loop resonant frequency) for the loop lengths and magnetic fields considered in this paper. A transmission coefficient of unity is achieved on resonance for all loops. Away from resonance, shorter loops (with higher frequency waves) transmit more energy between the chromosphere and the corona. The magnetic field variation is less significant. 

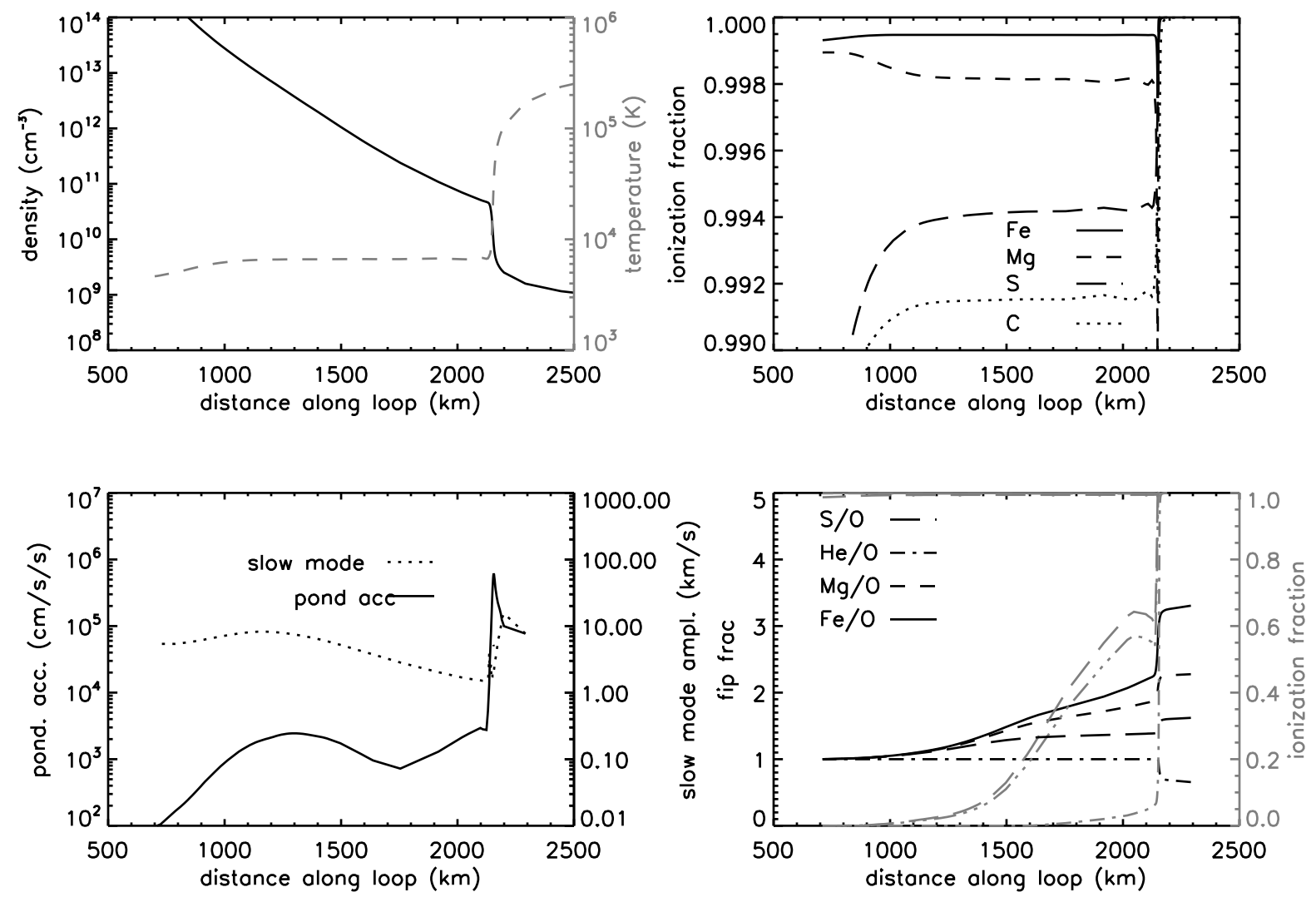

Fig. 7.- Chromospheric section of the model loop, length 75,000 km, magnetic field 15 $\mathrm{G}$, angular frequency $0.07 \mathrm{rad} \mathrm{s}^{-1}$. The upper left panel gives the density (solid line) and temperature (dashed line). Bottom left gives the ponderomotive force and slow mode wave (i.e. longitudinal wave excited by the Alfvén waves) amplitude. Upper right; ionization fractions of $\mathrm{Fe}, \mathrm{Mg}, \mathrm{S}$ and $\mathrm{C}$. Bottom right; fractionations of $\mathrm{Fe}, \mathrm{Mg}, \mathrm{S}$, and $\mathrm{He}$, as well as the ionization fractions in grey of $\mathrm{H}$ (dash), $\mathrm{C}$ (dash triple dot), and He (dash dot). 

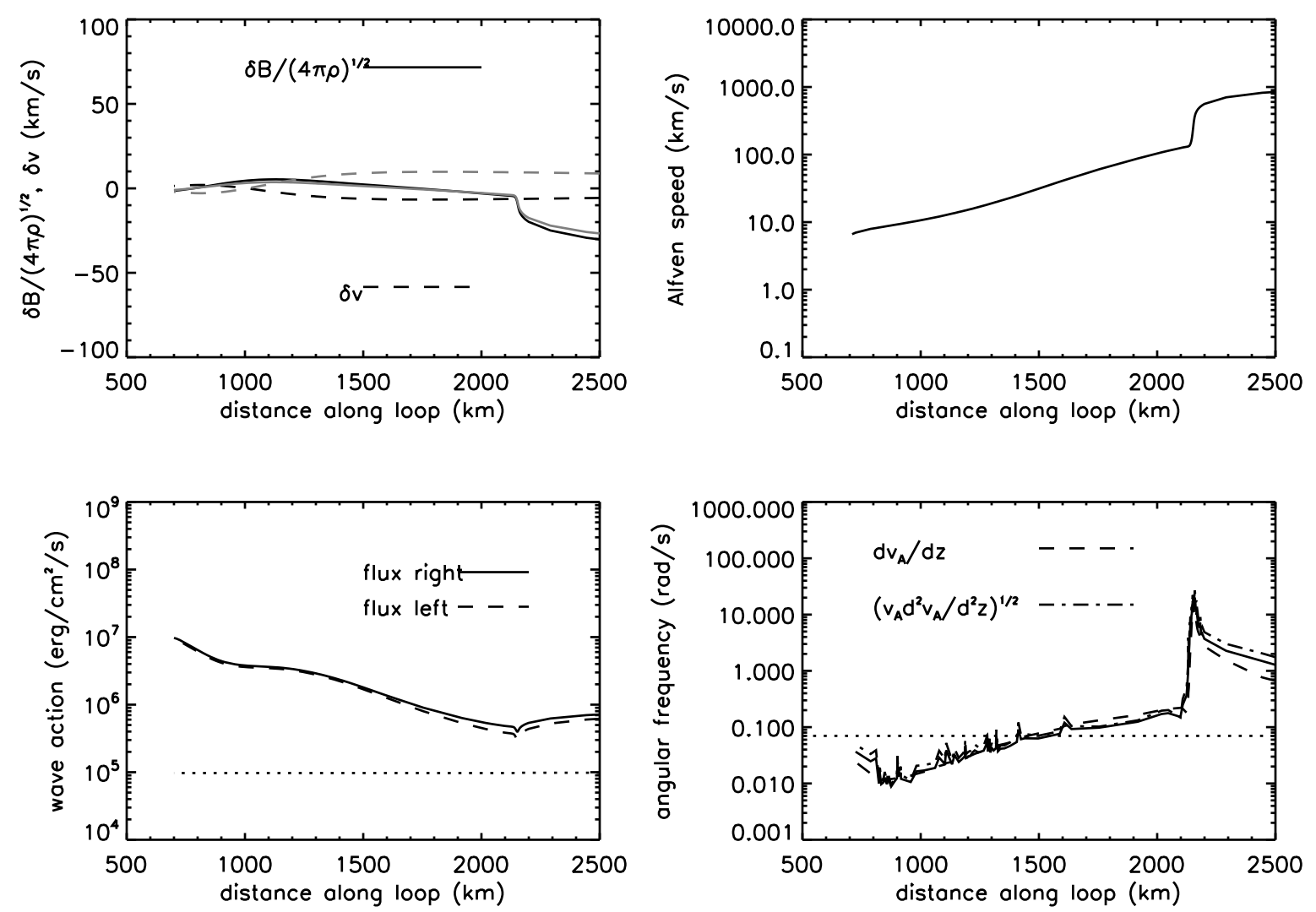

Fig. 8.- Chromospheric section of the model loop, length 75,000 km, magnetic field 15 $\mathrm{G}$, angular frequency $0.07 \mathrm{rad} \mathrm{s}^{-1}$. The upper left panel gives the amplitudes of Elsässer variables in $\mathrm{km} \mathrm{s}^{-1}(\delta B / \sqrt{4 \pi \rho}$ solid lines, $\delta v$ dashed lines, real parts in black and imaginary parts in gray.) At lower left are shown the wave energy fluxes in erg $\mathrm{cm}^{-2} \mathrm{~s}^{-1}$ for left and right going waves. The thin dotted line shows the difference in energy fluxes divided by the magnetic field strength and should be a horizontal line if energy is conserved. Upper right gives the Alfvén speed $V_{A}$ profile through the chromosphere. The bottom right right gives the variation of $\partial V_{A} / \partial z$ (dashed line) and $\sqrt{V_{A} \partial^{2} V_{A} / \partial z^{2}}$ (dot-dashed line). The solid line gives $\sqrt{\left(\partial V_{A} / \partial z\right)^{2} / 4+\left|V_{A} \partial^{2} V_{A} / \partial z^{2}\right| / 2}$, which is the Alfvén wave cutoff frequency as given by Moore et al. (1991) and Musielak et al. (1992). 


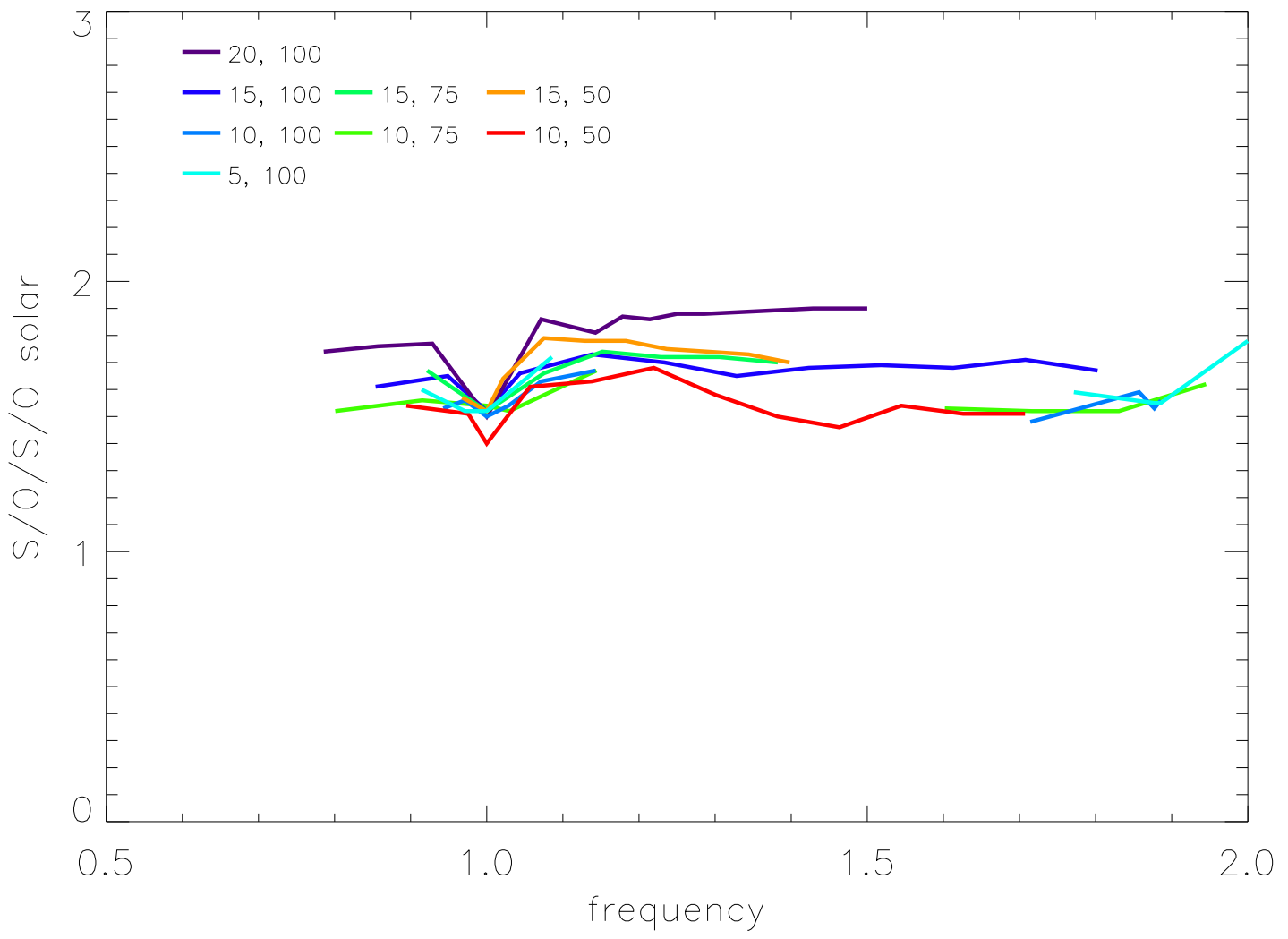

Fig. 9.- S/O relative to their solar ratio as a function of the frequency divided by the resonance frequency, similar to Figure 4. 


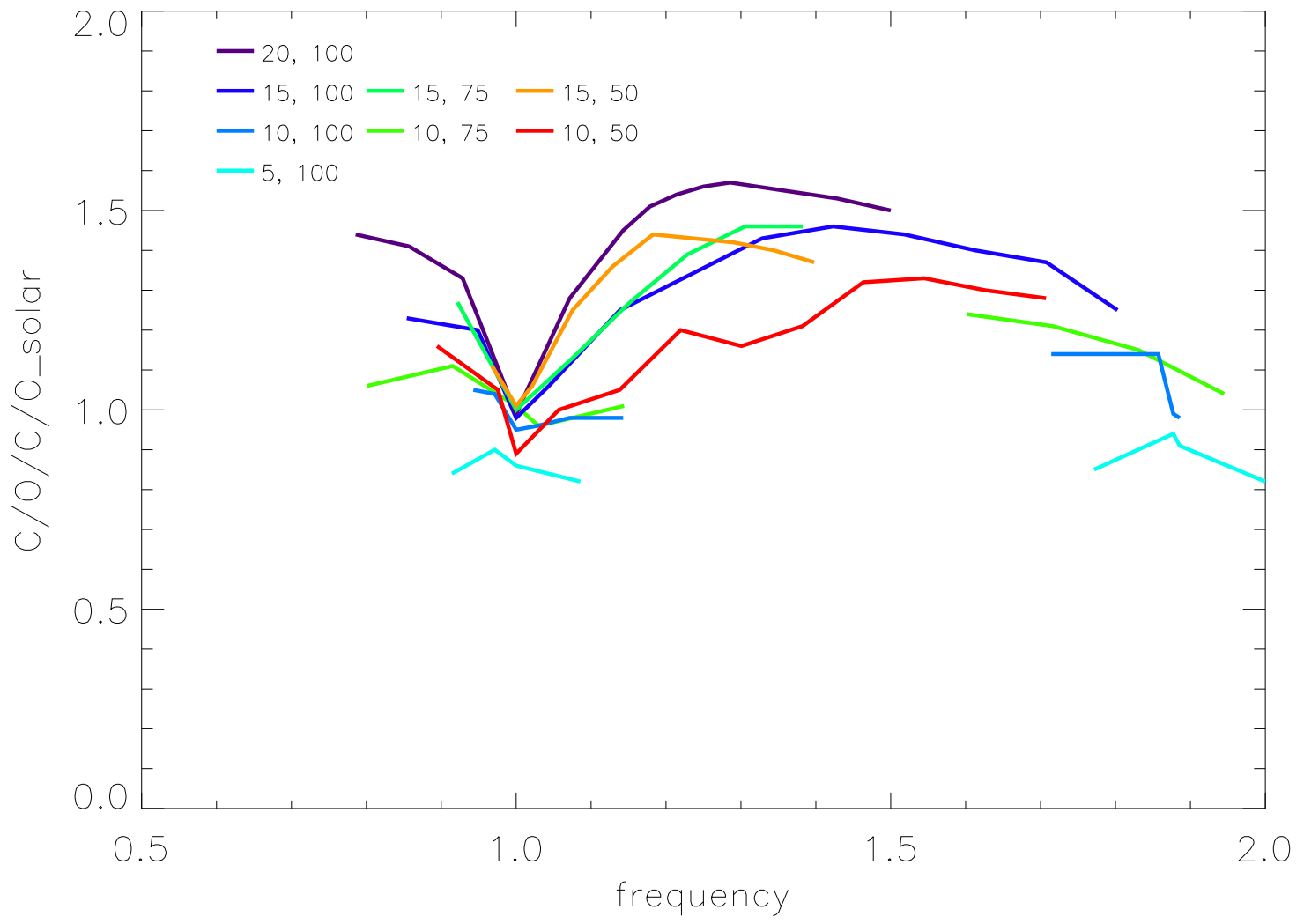

Fig. 10.- $\mathrm{C} / \mathrm{O}$ relative to their solar ratio as a function of the frequency divided by the resonance frequency, similar to Figure 4. 


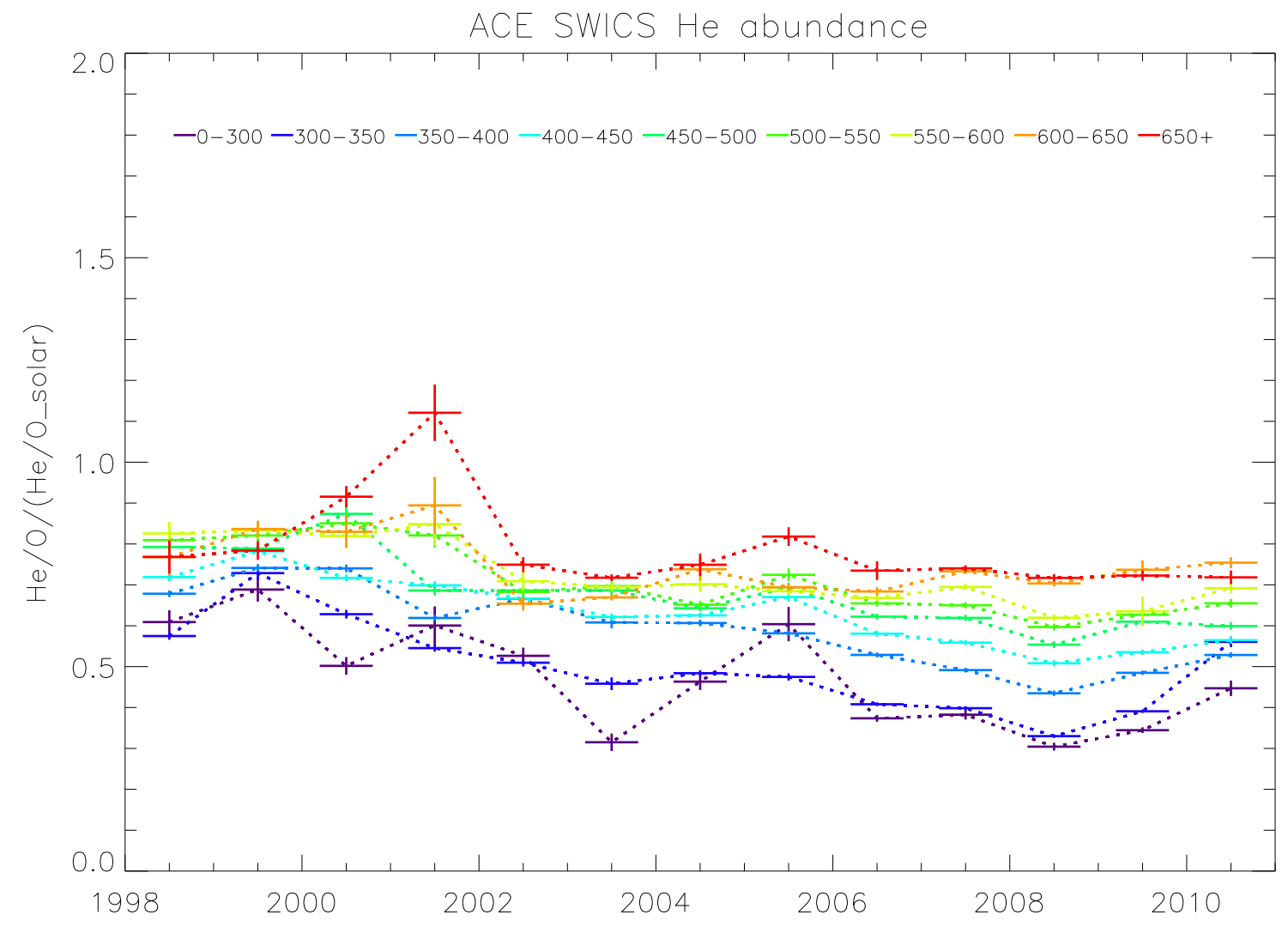

Fig. 11. - ACE SWICS yearly average of the measured $2 \mathrm{hr}$ averaged He/O ratio relative to photospheric (126) from 1998 to 2010 as grouped by He velocity in $50 \mathrm{~km} \mathrm{~s}^{-1}$ bins. Errors are the standard error on the mean. A trend of higher $\mathrm{He} / \mathrm{O}$ abundance ratio with higher solar wind speed is clearly visible, though strong variation with phase of the sunspot cycle is not seen. 


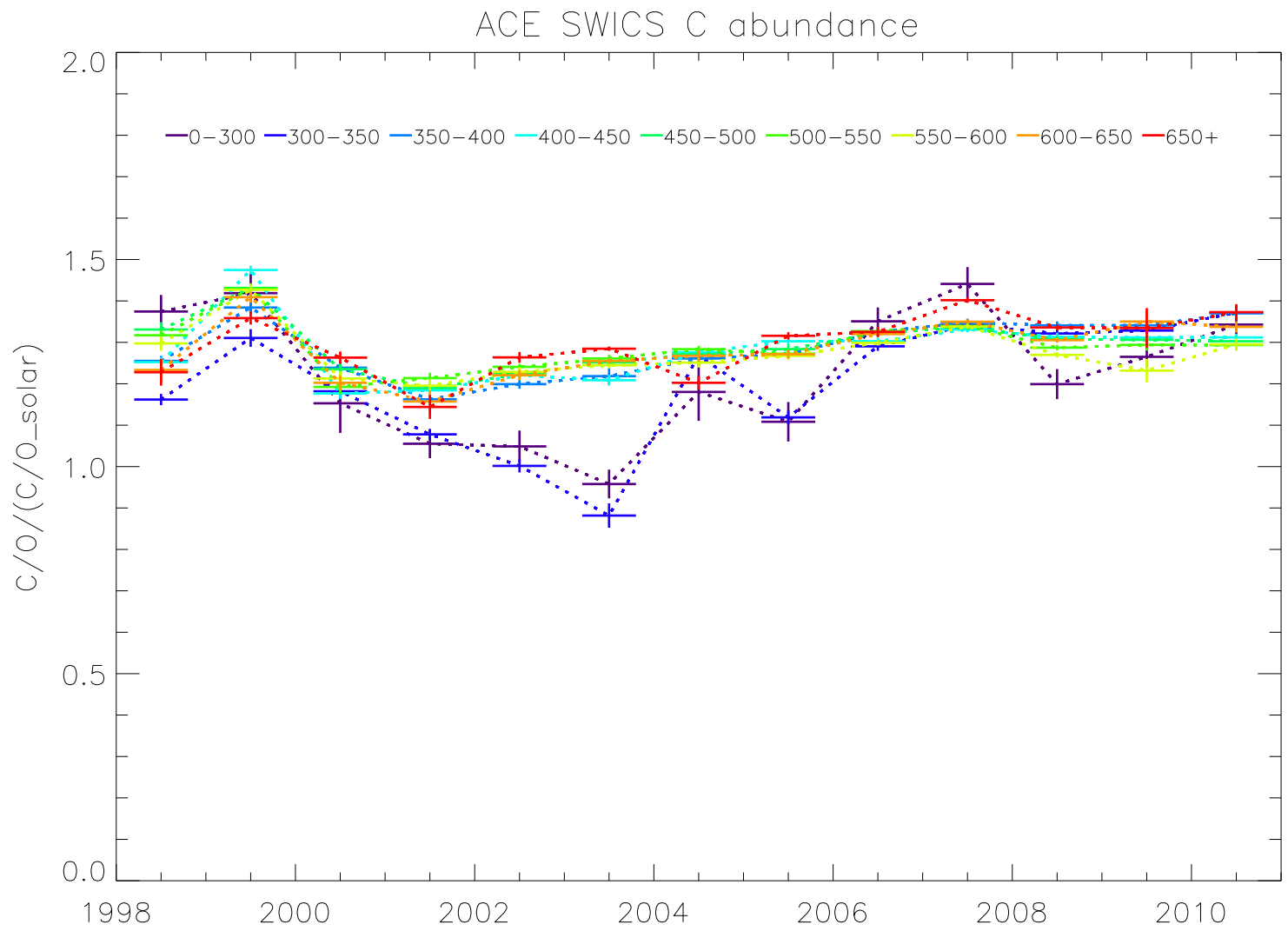

Fig. 12.- Same as Figure 11, but for $\mathrm{C} / \mathrm{O}$ relative to photospheric (0.550). A similar correlation of $\mathrm{C} / \mathrm{O}$ with wind speed is seen, especially around solar minimum years 2002 2004, and 2006. 